\title{
Analysis on Operation Risk of Continuous Sunken Tunnel on Expressway
}

\author{
Sheng ZHAO ${ }^{\text {a }}$, Yijia WANG ${ }^{\text {a }}$, Yujian XI ${ }^{\text {b,1 }}$, Yangyuyu XIA ${ }^{c}$ and Jiaming LU $^{\text {a }}$ \\ ${ }^{a}$ Guangzhou Guangming Expressway Co., Ltd, Guangzhou 510900, China \\ $\mathrm{b}$ School of Civil Engineering, Chongqing Jiaotong University, Chongqing 400074, \\ China \\ ${ }^{\mathrm{c}}$ China Merchants Chongqing Communications Research \& Design Institute Co. Ltd, \\ Chongqing 400067, China
}

\begin{abstract}
Based on the number of 519 tunnel risk incidents that occurred in the two tunnels of the Qifu Tunnel and the Zhongcun Tunnel during the period from 2018 to 2019 of Guangming Expressway, this paper studies the temporal and spatial characteristics and the risk distribution of the sunken continuous tunnel during the operation period of the expressway. Type of risk event. The results show that January, May, June and September of the year, as well as 14:00-16:00 and 16:00-18:00 during the day are periods of high tunnel risk; at the entrance section of continuous tunnels, Compared with other locations, the number of risk events in the transition section and the open section with sudden environmental changes and gradient changes is more; the types of risk events include safety hazards, roadblocks, vehicle failures, rear-end collisions, and equipment failures. The main types are vehicle failures. There are certain differences in the east-west direction. There are more vehicle breakdowns in the east-bound direction, more roadblocks in the west-bound direction, and more rear-end collisions in the eastbound direction. The main types of risk events are cars and trucks. Both cars and trucks have major risk event types. It is a vehicle failure. In rear-end collisions, small cars account for $65 \%$ of the risk models; risk identification methods include gun patrol discovery, road administration reporting, etc., of which gun patrol discovery is the most important identification method, accounting for $65 \%$ of the total. Through the analysis of the risk event characteristics of the sunken continuous tunnel of the expressway, it provides reference opinions for perfecting the research deficiencies in related fields in our country.
\end{abstract}

Keywords. Continuous tunnel, risk event, safety, risk identification

\section{Introduction}

As of the end of 2019, there were 19,067 road tunnels nationwide with a total length of 18.9666 million meters, of which 1,175 were extra-long tunnels with a total length of $5,217,500$ meters. The tunnel is a narrow and closed space structure. It plays an important role in shortening the driving distance, saving travel time, and protecting the ecological environment. However, due to its own characteristics, once an emergency occurs, the severity of the risk consequences is much greater than that of the tunnel. Outer ordinary section [1-3].

\footnotetext{
${ }^{1}$ Yujian Xi, School of Civil Engineering, Chongqing Jiaotong University, Chongqing 400074, China; E-mail:1056144397@qq.com.
} 
The construction of road tunnels in my country has only started in the 1980 s, so the research time on tunnel operation, accident handling and rescue is short, and experience is lacking [4]. Studying the risk and accident characteristics of highway tunnel sections can reduce casualties and economic losses. It is of great significance to effectively formulate tunnel safety measures and ensure the safety of tunnel operations $[5]$.

Corresponding research has been carried out on the risk of expressway operation. Zhao Feng and others conducted accident statistics analysis on highway tunnel operation accidents, and obtained the laws of accidents in form, cause, frequency and loss [6]; Zhang Shengrui took the tunnel group of Beijing-Zhuhai Expressway as an example to carry out the statistics of tunnel group traffic accidents According to analysis, it is found that the main area of accidents in the tunnel group is the entrance and exit of the tunnel. At the same time, the connecting section between the tunnels is also the area with a high incidence of accidents [7]; Ni Hongliang and others studied the distribution law of traffic accidents in the tunnel section of the Xihan Expressway, and proposed safety management measures for tunnel operations [8]. However, current research focuses on the operational risks of highway tunnels, and there is still a lack of research on the operational risks of the characteristics of highway tunnels.

This article analyzes the operation accidents of the Qifu Tunnel and Zhongcun Tunnel of Guangming Expressway from 2018 to 2019, and uses statistical comparison analysis methods to try to grasp the operating risk characteristics of the highway sunken continuous tunnel, and provide support for tunnel risk prevention and control. And perfect our country's risk research during the operation of the continuous sunken highway tunnel.

\section{Project Overview and Data Statistics}

The Qifu-Zhongcun continuous sinking tunnel is a typical highway sinking continuous tunnel located on the Guangzhou-Foshan (Gaoming) Expressway (Guangming Expressway), with a design speed of $100 \mathrm{~km} / \mathrm{h}$ and a two-way six-lane setting.

The route of the Qifu Tunnel basically follows Jinshan Avenue in Panyu District, and after passing through Shiguang Road, it overlaps with the Zhongping Crossroad and heads east to west. The total length is $1848 \mathrm{~m}$, of which, the open section is $240 \mathrm{~m}$, the closed section is $1178 \mathrm{~m}$, and the patio section is $430 \mathrm{~m}$. The route of the Zhongcun Tunnel is located on the south side of Zhongping Road in Panyu District, on the north side of Dafu Mountain Forest Park, and runs east to west. The total length is $920 \mathrm{~m}$, of which, the open section is $460 \mathrm{~m}$, the closed section is $240 \mathrm{~m}$, and the fully enclosed sound barrier section is $220 \mathrm{~m}$. It is currently a relatively rare large-scale open-cut and dark-buried highway urban sinking tunnel in China.

For the statistical data, we screened, the Qifu Tunnel starts at AK19+087.845 and ends at BK20+930; Zhongcun Tunnel starts at RK21+200 and ends at RK22+120, in order to ensure that the data is true and the source of the data is both For the data in the tunnel, the tunnel data calculated by K19, K20, and K21 are included in the data of this analysis. A total of 519 tunnel risk incidents were counted, including the date, time of occurrence, road section, location number, driving direction, spotting party, and brief description of abnormal conditions for each accident data. Among them, 314 were vehicle failures, 113 roadblocks, and potential safety hazards. 58 incidents, 32 rear-end collisions, and 2 equipment failures. 


\section{Distribution Characteristics of Tunnel Operation Risk}

\subsection{Time Distribution Characteristics of Operational Risk}

\subsubsection{Monthly Distribution Characteristics}

According to the statistical data of the risk events of the Qifu Tunnel and the Zhongcun Tunnel from January 2018 to December 2019, the distribution of the number of risk events in each month is drawn, as shown in figure 1.

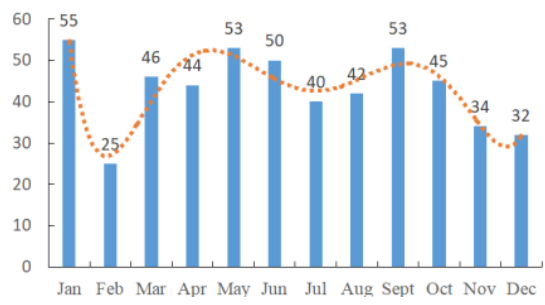

Figure 1. Monthly distribution characteristics of tunnel risk events.

It can be seen from figure 1 that the monthly distribution of risk events in Guangming Expressway Qifu Tunnel and Zhongcun Tunnel is wave-shaped. There are three peak periods for tunnel risks, namely, January, May, June and September. In February, there were at least 25 tunnel risk events. To sum up the reasons, January is during the Spring Festival transport period and the traffic volume is large. May, June and September have more holidays, such as Qingming Dragon Boat Festival and MidAutumn National Day, and the traffic volume is also large, so there are more tunnel risk events than other months; February is the Spring Festival, and most people spend the holidays at home, the traffic volume is small, and the risk of occurrence is low.

\subsubsection{Hourly Distribution Characteristics}

According to the number of risk events in the Qifu-Zhongcun tunnel from January 2018 to December 2019, the distribution map of the number of risk events in each time period is drawn. In order to better observe and study the number of risk events and the hourly regularity, the 24 hours a day is divided into two hours for segmentation, as shown in figure 2.

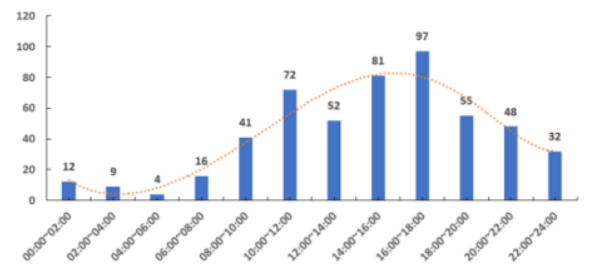

Figure 2. Time distribution characteristics of tunnel risk.

It can be seen from figure 2 that the number of risks in the tunnel changes in a cyclical wave line. After 6:00 in the morning, as people travel, the traffic volume gradually increases, and the risk events gradually increase. Between 16:00 and 18:00 in 
the afternoon (this time A total of 97 cases occurred in the segment, accounting for $19 \%$ ), reached the peak, and then gradually decreased, and the number of trips decreased in the evening, reaching the lowest point between 04:00 and 06:00 (a total of 4 cases occurred during this time period, accounting for $0.7 \%$ ). The hourly distribution of accidents is related to people's daily work and rest, physiological activities, natural environment, and fatigue degree, which has a certain regularity. At night, most people are still asleep, the traffic volume is small, and the number of tunnel risk events is small. During the day, the traffic volume increases, and the risk of tunnel risks increases. In the afternoon, drivers are prone to fatigue and sleepiness, which can easily cause fatigue driving, which increases the probability of tunnel risks.

\subsection{Spatial Distribution Characteristics of Operational Risk}

\subsubsection{Eastward Paragraph Features}

For every two hundred meters of the sunken continuous tunnel, the middle section between the two tunnels is $\mathrm{k} 20+930 \sim \mathrm{k} 21+200$, and statistics are made. For the convenience of statistics, K19+087 $\sim \mathrm{K} 19+300, \mathrm{~K} 20+700 \sim \mathrm{K} 20+930$ and $\mathrm{K} 21+800 \sim \mathrm{K} 22+120$ are all regarded as one segment. Because of the equipment failure, there is nothing to do, so they will not be included in the statistics for the time being, as shown in figure 3 . The arrow represents the driving direction.

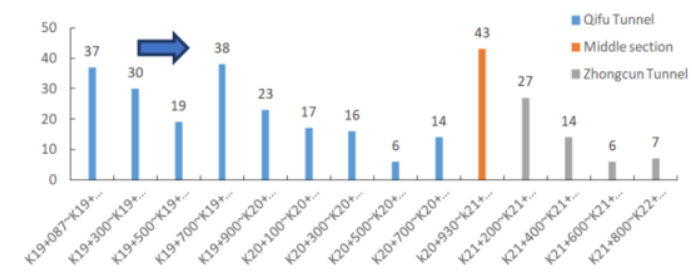

Figure 3. Statistics on the number of risks in the eastbound direction of the tunnel.

It can be seen from figure 3 that the risk event is higher at the entrance section of the Qifu Tunnel and gradually decreases. After a sudden point in the section $\mathrm{k} 19+700 \sim \mathrm{k} 19+900$, it continues to decrease until the middle section $(\mathrm{k} 20+930 \sim \mathrm{k} 21+200)$ reaches The highest, it gradually decreases after entering the Zhongcun Tunnel. There are many risk events in the entrance section of the tunnel. Due to the black hole effect, it is easy to make the driver nervous and increase the risk. The number of risks at $\mathrm{k} 19+700 \sim \mathrm{k} 19+900$ is prominent. The main reason is that this section is an open section in the tunnel. The driving environment Sudden change, the tunnel lighting has a large difference between inside and outside, and this section is near a variable slope point, with a gradient difference of $1.249 \%$; the middle section $(\mathrm{k} 20+930 \sim \mathrm{k} 21+200)$ has the highest number of risk events, mainly due to sudden changes in the driving environment, such as rain and snow. The road surface in the tunnel is good, but the road outside the tunnel is bad, and the vehicle is prone to slipping. In addition, the entrance and exit of the sunken tunnel are often up and down. The slope will also bring difficulties to the driver, and the risk probability It will be very high. The main risk types at the entrance section of Qifu Tunnel, the entrance section of Zhongcun Tunnel, and the middle section are vehicle failures [9]. 


\subsubsection{Westward Paragraph Features}

The analysis method is the same as that of the east direction. Draw the following statistical graph. The arrow represents the driving direction.

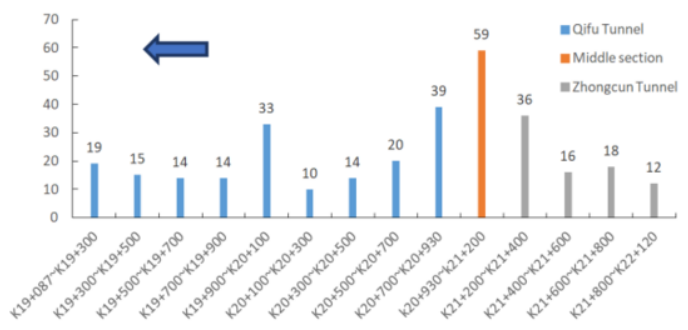

Figure 4. Statistics on the number of risks in the westbound direction of the tunnel.

It can be seen from figure 4 that from the direction of traffic, the risk event is low at the entrance section of Zhongcun Tunnel and remains stable until the exit section rises and reaches the highest in the middle section $(k 20+930 \sim k 21+200)$, and then enters the Qifu Tunnel. The entrance section continues to decline, and there is a sudden point in the $\mathrm{k} 19+700 \sim \mathrm{k} 19+900$ section until the number of risk events at the exit of the tunnel stabilizes. The entrance section of the westbound section of Zhongcun Tunnel is less than that of the eastbound section, mainly because the section is a semi-open section, with better light environment and lower risk probability; the number of risks at positions $\mathrm{k} 19+700 \sim \mathrm{k} 19+900$ is prominent, and this section is a tunnel The driving situation of the open section inside the tunnel is the same as the eastbound; the exit section of the Zhongcun Tunnel has a higher risk, mainly because the lighting environment inside and outside the tunnel changes suddenly, which is easy to cause the driver to have a white hole effect, and the risk is the highest in the middle section. The main risk is The type is vehicle failure, which is mainly due to sudden changes in the driving environment, which makes the number of risk events in this segment the highest.

\section{Analysis of Types of Risk Events in Tunnel Operation}

\subsection{Risk Event Type Analysis}

The passage of vehicles in the tunnel is complicated, and the types of risks are also diverse. The distribution of risk types is shown in figure 5.

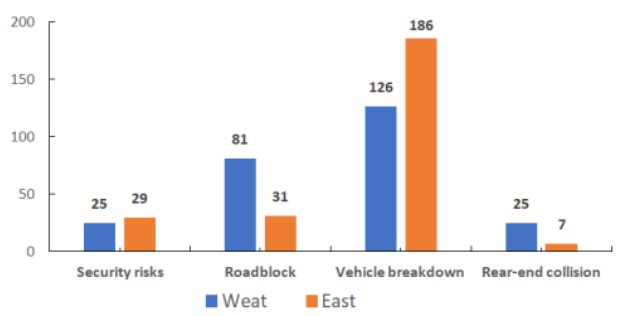

Figure 5. Types of tunnel risk events in the east-west direction. 
As can be seen from the above figure 5, according to the nature of the risk and the main body of the occurrence, it is divided into five types: safety hazards, vehicle failures, roadblocks, rear-end collisions, and equipment failures (because equipment failures have nothing to do with them, they are not listed here). The main types of risks in the east-west direction are vehicle failures, followed by roadblocks, hidden safety hazards and rear-end collisions. However, there are obvious differences in their specific numbers. There are 50 more roadblocks in the westbound direction than in the eastbound direction; the eastbound direction of the vehicle breakdown tunnel is 60 more than the westbound direction. It is worth noting that rear-end collisions mostly occur in the westbound direction of the tunnel, including 25 rear-end collisions in the westbound direction and 7 rear-end collisions in the eastbound direction of the tunnel. Westbound is 3.5 times more than the eastbound direction.

Analyze the causes of tunnel safety risks caused by potential safety hazards, roadblocks, vehicle breakdowns, and rear-end collisions, and get the following three statistical graphs.

Analyzing the types of potential safety hazards, the statistical chart shown in figure 6 is obtained. Among them, non-motor vehicles, pedestrians, and motorcycles are uncontrollable factors, accounting for $37.9 \%$. Tunnel water seepage, equipment damage, and irregular construction are controllable factors, accounting for $43.1 \%$. Vehicle stays, vehicle accidents and traffic jams are accidental factors, accounting for $19 \%$. Controllable factors are still in the dominant position, and maintenance needs to be strengthened to reduce risks.

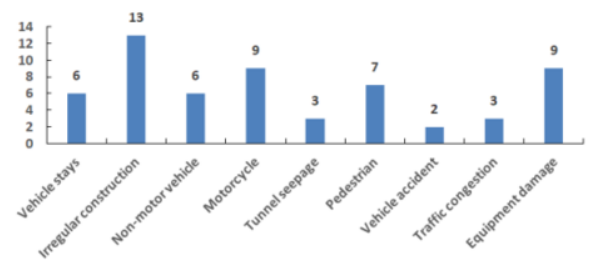

Figure 6. Types of safety hazards.

Analyze the types of roadblocks to get a statistical chart as shown in figure 7 . The main types of roadblocks include warning barrels, tire skins, wooden blocks (iron skin) and others, with 17 incidents, 19 incidents, 16, 12 incidents, and 20 incidents respectively.

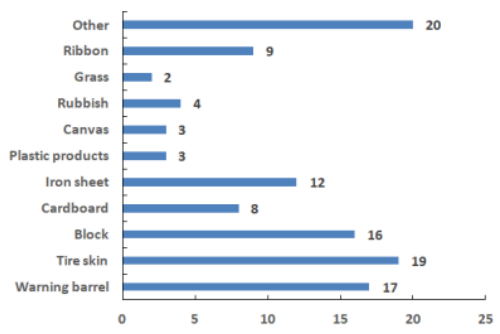

Figure 7. Types of roadblock risk.

Analyzing the types of vehicle failures, the statistical chart shown in figure 8 is obtained. The main types of vehicle failures are mechanical failures, with 100 cases, 
accounting for $47.8 \%$; followed by puncture, with 56 cases, accounting for $26.8 \%$; There were 18 and 14 electricity cases, accounting for $8.6 \%$ and $6.7 \%$ respectively.

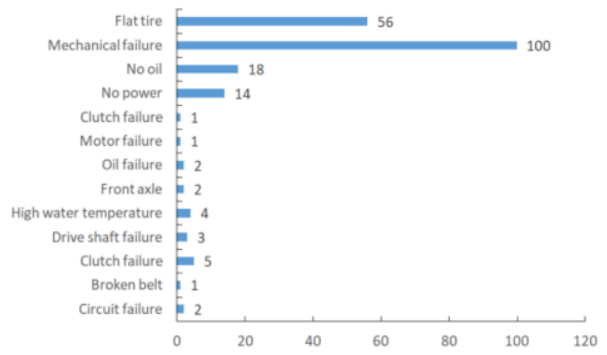

Figure 8. Types of vehicle failure risk.

There were a total of 32 rear-end collisions, of which car models accounted for the most, accounting for $65 \%$; followed by passenger cars, accounting for $20 \%$; trucks, $14 \%$; vans, $1 \%$.

\subsection{Analysis of Tunnel Risk Models}

Then count the vehicles involved in the 519 risk events, and classify these vehicles into cars, trucks, buses, vans and other models, as shown in figure 9.

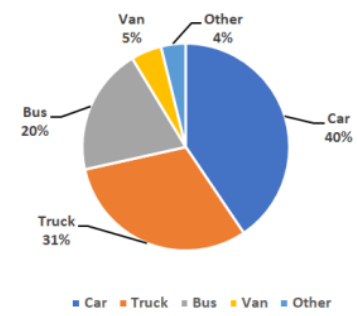

Figure 9. Distribution of risk vehicles in tunnels.

It can be seen from figure 9 that the risk models of road tunnels are mainly trucks and cars, which account for $31 \%$ and $40 \%$ of the total models, and $71 \%$ of the accident models.

Studies have shown that the risk events of the highway sunken continuous tunnel are mainly cars and trucks. The main risk events of trucks in the sunken high-speed continuous tunnel are vehicle failures. Mechanical failures and punctures are common occurrences of trucks. Risk events. The main risks of small cars are vehicle breakdown and rear-end collision [10].

\section{Risk Identification and Analysis of Tunnel Operation}

According to the statistics, there are currently eight methods of tunnel risk detection, namely, highways report, gun patrol discovery, Gao $\mathrm{Wu}$ call, squad leader report, 
traffic police notice, toll station report, driver call, and cooperative unit report. And draw the following statistical chart.

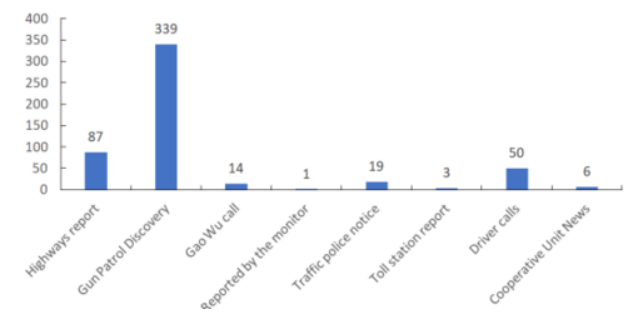

Figure 10. Statistics of types of tunnel risk discoverers.

It can be seen from figure 10 that gun patrol discovery is the main method of tunnel risk discovery, with a total of 339 cases, accounting for $65 \%$ of the total; followed by road administration reports 87 cases, accounting for $17 \%$ of the total; driver calls and traffic police notifications were 50 cases and 19 cases respectively. The total number of cases, accounting for $9.6 \%$ and $3.7 \%$ of the total; the final report from the cooperation unit, toll booth, and squad leader were 6, 3, and 1 respectively. As the main method of discovery, gun patrol discovery reflects the progress of technical means. Discovery methods have become intelligent, and the proportion of manual discovery methods will decrease. The discovery of tunnel risks also requires manual assistance and social forces, so other methods of discovery besides gun patrols are also important methods of discovery.

Count the discoverers of tunnel risk types. The identification methods are divided into gun patrol discovery, road administration report, and others. Others include Gao $\mathrm{Wu}$ calls, squad leader reports, traffic police notifications, toll booth reports, driver calls, and cooperative unit reports. Because the two cases of equipment failure were reported by the road administration and the gun patrol, we will not make statistics here this time, and draw the statistics of the data that have been obtained as follows.

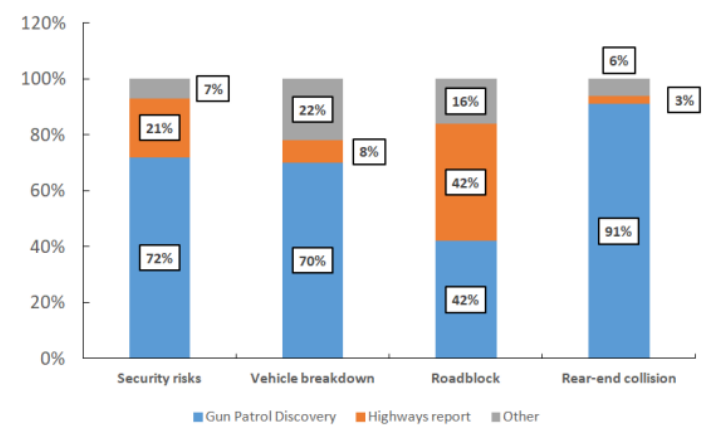

Figure 11.Tunnel risk discovery sender.

It can be seen from figure 11 that gun patrol discovery is the main method for detecting parties of each type of risk. In rear-end collisions, gun patrol discovery is the main method, accounting for $91 \%$ of 32 accidents; Among vehicle failures, gun patrols also accounted for $70 \%$ of the total discoveries; gun patrols for safety hazards accounted for $72 \%$; Gun patrols for roadblock risks accounted for $42 \%$, and road administration reports accounted for $42 \%$. There are many ways to discover risks. Gun 
patrol discovery, as the main discovery method, should be focused on. At the same time, the coexistence of various other discovery methods, complements each other, and discovers tunnel risks in a more timely and effective manner.

\section{Risk Response Measures Recommendations}

The sunken continuous tunnel of the expressway has the same risk characteristics as the ordinary tunnel, but the sunken expressway tunnel has its own characteristics in the risk characteristics due to its own structural characteristics, so when dealing with the risks of the tunnel, it is necessary to Take risk response measures based on its characteristics.

(1) Since the entrance and exit of the sunken tunnel are downhill and uphill respectively, in the case of sudden changes in the light environment at the entrance and exit, the road gradient has been changed and the driving difficulty has been increased. Therefore, in addition to setting up warning signs at the entrance and exit, In addition, the adhesion coefficient of the road surface of the tunnel should be improved to prevent the vehicle from slipping [11].

(2) For a continuous sunken highway tunnel, there will be a slope point in the tunnel. The size of the slope point will also affect the safety of vehicles. Therefore, when designing this type of tunnel, the slope difference of the slope point should be avoided as much as possible, and the key point of the slope point section should be monitored in future operation and maintenance [12].

\section{Conclusion}

At present, there are few domestic researches on the continuous sunken tunnels of expressways. In this risk analysis of the Qifu Tunnel and Zhongcun Tunnel on the Guangming Expressway, we mainly focus on the time distribution characteristics, spatial distribution characteristics, and operational risk factors of the tunnels. And risk identification to analyze the four aspects.

(1) The number of risks in the tunnel is usually 14:00-16:00, 16:00-18:00 in January, May, September, October and one day.

(2) The entrance section, transition section, exit section and k19+700 k19+900 sections of the continuous sunken highway tunnel have more risk events than other locations.

(3) There are five types of tunnel risk events in the survey, mainly vehicle failures, then roadblocks and hidden safety hazards, and finally rear-end collisions and equipment failures. The main cause of vehicle failure is mechanical failure. The main safety hazards are irregular construction and equipment problems. The roadblocks are mainly tire skins and some other sundries.

(4) In all risk events, cars and trucks accounted for the largest proportion, accounting for $40 \%$ and $31 \%$, respectively, followed by passenger cars and vans, accounting for $20 \%$ and $5 \%$, respectively, and other types of vehicles accounting for $4 \%$.

(5) Risk identification mainly includes gun patrol discovery, accounting for $65 \%$ of the total; followed by road administration reports and driver calls, accounting for $17 \%$ and $10 \%$ of the total; then traffic police notifications and Gao $\mathrm{Wu}$ calls, accounting for $4 \%$ and $3 \%$ of the total; finally There were only 6 reports, 3 reports, and 
1 report from the cooperating unit, the toll station, and the monitor. As the main detection method, gun patrol found $70 \%$ of vehicle failures, $72 \%$ of safety hazards, $41 \%$ of roadblocks and $90 \%$ of rear-end collisions, and both equipment failures were discovered by gun patrols. The main means of identifying roadblocks is road administration reporting, accounting for $48 \%$.

\section{References}

[1] Yao ChY. Research on construction technology of large span and small clear distance tunnel. Engineering and Technological Research. 2021 May; 6(3): 86-87.

[2] Zhang GZh, Cui ShD, Zhang HW, Xu MF. Impact of tunnel engineering on ecological environment and environmental effects. Journal of geological Hazards and Environmental Preservation. 2017 Dec; 28(4): 53-55.

[3] Wang Q, Jiang Sh P, Liu H L, Ma F. Analysis on Traffic accident characteristics of urban road tunnels Chinese Journal of Underground Space and Engineering. 2019 Aug; 15(S1): 460-469.

[4] Zhang LY, Qiao Zh, Huang Y. Analysis of reasons of traffic accidents in Chengdu-Chongqing Expressway Chongqing Section and study on treatment measures. Traffic Technology of Highway and Transport. 2008 Feb; 2(1): 121-123.

[5] Hu LW, Chen Zh, Zhang T. Study on car-following characteristics and safety based on extra-long expressway tunnel section in plateau region. Journal of Highway and Transportation Research and Development. 2018 Jan; 35(1): 112-114.

[6] Zhao F, Xia YX, Xie T. Statistical analysis of road tunnel operation accidents Highwa. Highway. 2014 Jun; 59(06): 280-287.

[7] Zhang ShR, Ma ZhL, Shi Q. Distribution characteristics and countermeasures of traffic accidents in expressway tunnel group. Journal of Chang'an University (Natural Science Edition). 2007 Jan; (01): 63 66.

[8] Ni HL, Dai YH, Zhao QX. Study on the distribution of highway tunnel accidents. Highway. 2010 Apr; (04): 126-129.

[9] Wang BL, Ye Y, Liu C, et al. Application and experimental evaluation of natural light in tunnel lighting engineering. Modern Tunnelling Technology. 2014 Jun; 51(3): 35-42.

[10] Zhang YCh, He Ch, Fang Y, et al. Analysis on the causes of traffic accidents in highway tunnel groups. Chinese Safety Science Journal. 2009 Sept; 19(9): 120-124.

[11] Guo YH. Research on Highway Tunnel Safety and Accident Prevention Countermeasures. World of Transportation (late issue). 2020 Jun; (6): 152-153.

[12] Wang HM, Lan L, He C, et al. Research on truck driving risks and countermeasures in super-long downhill tunnels on expressway. Highway. 2020 Apr; 65(4): 232-237. 